\title{
Bubble stabilized discontinuous Galerkin methods on conforming and non-conforming meshes
}

\author{
Erik Burman · Benjamin Stamm
}

Received: 4 January 2010 / Accepted: 7 December 2010 / Published online: 13 January 2011

(C) The Author(s) 2011. This article is published with open access at Springerlink.com

\begin{abstract}
The aim of this paper is to discuss the properties of the bubble stabilized discontinuous Galerkin method with respect to mesh geometry. First we show that on certain non-conforming meshes the bubble stabilized discontinuous Galerkin method allows for hanging nodes/edges. Then we consider the case of conforming meshes and present a post-processing algorithm based on the Crouzeix-Raviart method to obtain the Bubble Stabilized Discontinuous Galerkin (BSDG) method. Although finally the post-processed solution does not coincide with the BSDG-solution in general, they satisfy the same (approximation) properties and are close to each other. Moreover, the post-processed solution has continuous flux over the edges.
\end{abstract}

Keywords Discontinuous Galerkin · Elliptic equation · Crouzeix-Raviart approximation

Mathematics Subject Classification (2000) $65 \mathrm{M} 160 \cdot 65 \mathrm{M} 15$

\section{Introduction}

Consider the following diffusion equation: find $u: \Omega \rightarrow \mathbb{R}$ such that

$$
\begin{cases}-\Delta u=f & \text { in } \Omega, \\ u=0 & \text { on } \partial \Omega,\end{cases}
$$

E. Burman

Department of Mathematics, University of Sussex, BN1 9RF Brighton, UK

e-mail: e.n.burman@sussex.ac.uk

B. Stamm ( $\varangle)$

Department of Mathematics, University of California, 970 Evans Hall, Berkeley, CA 94720, United States

e-mail: stamm@berkeley.edu 
where $f \in L^{2}(\Omega)$. Assuming a homogeneous boundary condition and a diffusion coefficient of one is not an essential restriction but allows us to simplify the presentation and focus on the key features of the analysis.

Discontinuous Galerkin method were first introduced for hyperbolic problems by Reed and Hill in [20] for neutron transport problems. It was further analyzed by Lesaint and Raviart [16] and Johnson and Pitkäranta [14]. It became popular for transport problems due to its good stability properties and is nowadays a established tool for hyperbolic problems.

For elliptic problems on the other hand the first papers include the work of Nitsche [18], Baker [3], Douglas and Dupont [13], Wheeler [22] and Arnold [2]. These contributions led to the Symmetric Interior Penalty (discontinuous) Galerkin (SIPG) method. Only in the nineties discontinuous methods became more popular and many variants such as the Non-symmetric Interior Penalty (discontinuous) Galerkin (NIPG) method [4, 15, 19, 21] or the Local Discontinuous Galerkin (LDG) method [5, 11] were introduced. One advantage of the DG-method compared to continuous FEM is the enhanced local conservation. However the local conservation depends on the numerical flux and thus on the stabilization parameter which may vary in space and depend on material properties for more complex problems. On the other hand it was also proven $[15,21]$ that the NIPG method does not need to be stabilized for polynomial degrees larger or equal than two. This variant is referred to as the Baumann-Oden method. This led to a further improved local mass conservation property, but due to the non-symmetry of the method, optimal $L^{2}$-convergence does not hold in general.

In 2006, Brezzi and Marini [6] then proved that the smallest discontinuous finite element space for which the NIPG method is stable without stabilization is a finite element space consisting of polynomials of degree one enriched locally by one bubble of degree two. Independently, Burman and Stamm [8, 9] introduced the symmetric version of the Bubble Stabilized Discontinuous Galerkin (BSDG) method and proved its stability and convergence. The numerical scheme uses the same bubble enriched finite element space as the one introduce by Brezzi and Marini, but its bilinear form is symmetric. More precisely, it uses the same bilinear form as the SIPG method but without penalty term. Thus stability is achieved through particular properties of the finite element space and not through penalty. This method combines the optimal $L^{2}$-convergence of a symmetric scheme and the improved local mass conservation property due to the absence of a penalty term.

In this paper we address two questions left open in [8, 9]: the question of hanging nodes/edges and the question of hierarchic construction of the DG solution.

Indeed an important advantage of a DG-method is the possibility to use hanging nodes. Since the BSDG-method uses the structure of the finite element space to obtain stability the introduction of hanging nodes/edges is no longer straightforward. In this paper we give sufficient conditions for stability of the BSDG-method on meshes with hanging nodes/edges. We only consider a very simple setting where a conforming base mesh can be refined in a way giving rise to hanging nodes. The extension to more general cases is non-trivial, in the framework of the symmetric BSDG-method and here the non-symmetric approach of [1] that is valid on general polygonal decompositions seems more promising thanks to the positivity of the involved bilinear form. 
In [8] we showed that the solution of the SIPG method using piecewise affine elements naturally decomposes into two components. One is the classical CrouzeixRaviart solution and the other is a perturbation that ensures local mass-conservation. It is indeed the constraint of local mass conservation that can leads to loss of stability. Similar results have recently been used for the design of optimal preconditioners for DG-methods [12]. In this paper we show a similar result for the BSDG-method.

Herein we propose an efficient hierarchic implementation of the BSDG-method that does not need more globally coupled degrees of freedom than the nonconforming Crouzeix-Raviart method. This approach consists of computing the Crouzeix-Raviart solution in a first step (global solve) and then to post-process it locally in the bubble enriched finite element space (local solves). The post-processed solution coincides with the BSDG solution only in the case of elementwise constant source term, but is always an approximation that is sufficiently close to satisfy the same convergence properties as the BSDG solution. The normal gradient of the postprocessed approximation is continuous over interelement faces. As a consequence, we present a numerical algorithm that satisfies the local mass conservation property exactly (since the flux is continuous across faces), does not need more globally coupled degrees of freedom than the Crouzeix-Raviart method and converges optimally in the $L^{2}$-norm. The method can be seen as an efficient solution algorithm for the BSDG-method, but also as a prototype parallel domain-decomposition preconditioner for discontinuous Galerkin methods.

This paper is structured as follows. In Sect. 2 we introduce and develop preliminary results needed for the later analysis. The different kind of numerical approximations are introduced in Sect. 3, where also an overview over the basic properties of the BSDG-method is given. Then in Sect. 4 we introduce sufficient conditions under which hanging nodes/edges are allowed and prove stability and optimal convergence results on such meshes.

As a "warm up exercise" and preparation for the following Section we propose a post-processing of the Crouzeix-Raviart solution to get the Raviart-Thomas approximation based on some earlier work of Marini [17] in Sect. 5. The main result is presented in Sect. 6 where the post-processing in the bubble enriched finite element space is introduced and analyzed. As a summary, the relation of all different approximations for piecewise constant data are investigated in Sect. 7.

\section{Preliminary results}

Let $\Omega$ be a polygonal domain (polyhedron in three space dimensions) in $\mathbb{R}^{d}, d=2,3$, with outer normal $n$. Let $\mathcal{K}$ be a subdivision of $\Omega \subset \mathbb{R}^{d}$ into non-overlapping $d$ simplices $\kappa$ and denote by $N_{\mathcal{K}}$ the number of simplices of the mesh. Suppose that each $\kappa \in \mathcal{K}$ is an affine image of the reference element $\widehat{\kappa}$, i.e. for each element $\kappa$ there exists an affine transformation $T_{\kappa}: \widehat{\kappa} \rightarrow \kappa$.

Let $\mathcal{F}_{i}$ denote the set of interior faces $((d-1)$-manifolds $)$ of the mesh, i.e. the set of faces that are not included in the boundary $\partial \Omega$. The set $\mathcal{F}_{e}$ denotes the faces that are included in $\partial \Omega$ and define $\mathcal{F}=\mathcal{F}_{i} \cup \mathcal{F}_{e}$. Further $\mathcal{F}(\kappa)$ denotes the set of faces of the element $\kappa$. Define by $N_{\mathcal{F}}=\operatorname{card}(\mathcal{F})$ and $N_{\mathcal{F}_{i}}=\operatorname{card}\left(\mathcal{F}_{i}\right)$ the number of faces 
resp. interior faces of the mesh. Denote by $\Gamma$ the skeleton of the mesh, i.e. the set of points belonging to faces, $\Gamma=\{x \in \bar{\Omega} \mid \exists F \in \mathcal{F}$ s.t. $x \in F\}$.

Assume that $\mathcal{K}$ is shape-regular, does not contain any hanging node and covers $\bar{\Omega}$ exactly. For an element $\kappa \in \mathcal{K}, h_{\kappa}$ denotes its diameter and for a face $F \in \mathcal{F}, h_{F}$ denotes the diameter of $F$. Set $h=\max _{\kappa \in \mathcal{K}} h_{\kappa}$ and let $h$ be the function such that $\left.\mathrm{h}\right|_{\kappa}=h_{\kappa}$ and $\left.\mathrm{h}\right|_{F} ^{\circ}=h_{\mathrm{F}}$ for all $\kappa \in \mathcal{K}$ and $F \in \mathcal{F}$.

For a subset $R \subset \Omega$ or $R \subset \mathcal{F},(\cdot, \cdot)_{R}$ denotes the $L^{2}(R)$-scalar product, $\|\cdot\|_{R}=$ $(\cdot, \cdot)_{R}^{1 / 2}$ the corresponding norm, and $\|\cdot\|_{s, R}$ the $H^{s}(R)$-norm. The element-wise counterparts will be distinguished using the discrete partition as subscript, for example $(\cdot, \cdot)_{\mathcal{K}}=\sum_{\kappa \in \mathcal{K}}(\cdot, \cdot)_{K}$. For $s \geq 1$, let $H^{s}(\mathcal{K})$ be the space of piecewise Sobolev $H^{s}$-functions and denote its norm by $\|\cdot\|_{s, \mathcal{K}}$.

In this paper $c>0$ denotes a generic constant and can change at each occurrence, while an indexed constant stays fix. Any constant is independent of the mesh size $h$.

Further let us define the jump and average operators. Fix $F \in \mathcal{F}_{i}$ and thus $F=\kappa_{1} \cap$ $\kappa_{2}$ with $\kappa_{1}, \kappa_{2} \in \mathcal{K}$. Let $v \in H^{1}(\mathcal{K}), w \in\left[H^{1}(\mathcal{K})\right]^{d}$ and denote by $v_{i}, w_{i}, i=1,2$, the restriction of $v$ resp. $w$ to the element $\kappa_{i}$, i.e. $v_{i}=\left.v\right|_{\kappa_{i}}$ resp. $w_{i}=\left.w\right|_{\kappa_{i}}$ and denote by $n_{i}$ the exterior normal of $\kappa_{i}$. Then we define the standard average and jump operators by

$$
\begin{aligned}
\{v\}=\frac{1}{2}\left(v_{1}+v_{2}\right), \quad[v]=v_{1} n_{1}+v_{2} n_{2}, \\
\{w\}=\frac{1}{2}\left(w_{1}+w_{2}\right), \quad[w]=w_{1} \cdot n_{1}+w_{2} \cdot n_{2} .
\end{aligned}
$$

On outer faces $F \in \mathcal{F}_{e}$ we define them by

$$
\{v\}=v, \quad[v]=v n, \quad\{w\}=w, \quad[w]=w \cdot n
$$

where $n$ is the outer normal of the domain $\Omega$. Observe that $[v](x),\{w\}(x) \in \mathbb{R}^{d}$ and thus we introduce also scalar quantities of the jump and average, that we index by $s$ :

$$
[v]_{s}=[v] \cdot n_{F}, \quad\{w\}_{s}=\{w\} \cdot n_{F},
$$

where $n_{F} \in\left\{n_{1}, n_{2}\right\}$ is arbitrarily chosen but fixed. Observe that also $[v]=[v]_{s} n_{F}$ and thus that $\|[v]\|_{\mathcal{F}}=\left\|[v]_{s}\right\|_{\mathcal{F}}$ and $\|[v]\|_{\mathcal{F}_{i}}=\left\|[v]_{s}\right\|_{\mathcal{F}_{i}}$. Moreover note that

$$
[v] \cdot\{w\}=[v]_{S}\{w\}_{S} .
$$

The following integration by parts formula holds (see for instance [2]).

Lemma 2.1 (Integration by parts formula) Let $v, w \in H^{2}(\mathcal{K})$, then

$$
(\nabla v, \nabla w)_{\mathcal{K}}=-(\Delta v, w)_{\mathcal{K}}+(\{\nabla v\},[w])_{\mathcal{F}}+([\nabla v],\{w\})_{\mathcal{F}_{i}}
$$

2.1 Bubble stabilized finite element space

Let us denote by $V_{h}^{p}$ the standard discontinuous finite element space of degree $p \geq 0$ defined by

$$
V_{h}^{p}=\left\{v_{h} \in L^{2}(\Omega)\left|v_{h}\right|_{\kappa} \in V_{h}^{p}(\kappa), \forall \kappa \in \mathcal{K}\right\}
$$


where $V_{h}^{p}(\kappa)$ denotes the set of polynomials of maximum degree $p$ on $\kappa$. Consider the locally defined enriched finite element space

$$
V_{b s}(\kappa)=V_{h}^{1}(\kappa) \oplus\left\{v_{h} \in L^{2}(\kappa) \mid v_{h}(x)=\alpha x \cdot x, \alpha \in \mathbb{R}\right\}
$$

and its global counterpart

$$
V_{b s}=V_{h}^{1} \oplus\left\{v_{h} \in L^{2}(\Omega) \mid v_{h}(x)=\alpha x \cdot x, \alpha \in V_{h}^{0}\right\}
$$

where $x=\left(x_{1}, \ldots, x_{d}\right)$ denotes the physical variable. Let us additionally define some functional space that consists of functions only defined on the skeleton of the mesh:

$$
W_{h}^{0}=\left\{v_{h} \in L^{2}(\Gamma)\left|v_{h}\right|_{F} \in \mathbb{R}, \forall F \in \mathcal{F}\right\} .
$$

Again, we define a local counterpart by

$$
W_{h}^{0}(\kappa)=\left\{v_{h} \in L^{2}(\partial \kappa)\left|v_{h}\right|_{F} \in \mathbb{R} \text {, for all faces } F \text { of } \partial \kappa\right\} .
$$

Let $v \in H^{1}(\mathcal{K})$ and define by $\overline{\{v\}}, \overline{[v]}_{S}$ the $L^{2}$-projection of $\{v\}$ resp. $[v]_{s}$ onto $W_{h}^{0}$, i.e.

$$
\begin{aligned}
& \left(\overline{\{v\}}, w_{h}\right)_{\mathcal{F}}=\left(\{v\}, w_{h}\right)_{\mathcal{F}}, \quad \forall w_{h} \in W_{h}^{0}, \\
& \left({\overline{[v]_{s}}}_{s}, w_{h}\right)_{\mathcal{F}}=\left([v]_{s}, w_{h}\right)_{\mathcal{F}}, \quad \forall w_{h} \in W_{h}^{0} .
\end{aligned}
$$

Further define $\overline{[v]}=\overline{[v]}_{S} n_{F}$.

We denote by $R T_{0}(\kappa)$ the local Raviart-Thomas space of order 0 defined by

$$
R T_{0}(\kappa)=\left[\mathbb{P}_{0}(\kappa)\right]^{d}+x \mathbb{P}_{0}(\kappa)
$$

The global Raviart-Thomas space and the Crouzeix-Raviart space are defined in a standard fashion by

$$
\begin{aligned}
& V_{r t}=\left\{v \in\left[L^{1}(\Omega)\right]^{d}|v|_{\kappa} \in R T_{0}(\kappa) \forall \kappa \in \mathcal{K},\left.[v]\right|_{F}=0, \forall F \in \mathcal{F}_{i}\right\}, \\
& V_{c r}=\left\{v \in L^{1}(\Omega)|v|_{\kappa} \in \mathbb{P}_{1}(\kappa) \forall \kappa \in \mathcal{K},\left.\overline{[v]}\right|_{F}=0, \forall F \in \mathcal{F}\right\} .
\end{aligned}
$$

Note that a particular property of the space $V_{b s}$ is that for any $v_{h} \in V_{b s}$ we have that $\left.\nabla v_{h}\right|_{\kappa} \in R T_{0}(\kappa)$, c.f. [8]. Thus it follows that $\left[\nabla v_{h}\right]=\overline{\left[\nabla v_{h}\right]}$ and $\left\{\nabla v_{h}\right\}=\overline{\left\{\nabla v_{h}\right\}}$ for all $v_{h} \in V_{b s}$ on all faces. Finally let us introduce the following local norm

$$
\mid] v\left[\left.\right|_{\mathcal{K}} ^{2}:=\|\nabla v\|_{\mathcal{K}}^{2}+\left\|h^{-\frac{1}{2}} v\right\|_{\partial \kappa}^{2}\right.
$$

for all $v \in H^{1}(\kappa)$ and $\kappa \in \mathcal{K}$. On a global level we define

$$
\|v\|^{2}:=\|\nabla v\|_{\mathcal{K}}^{2}+\left\|\mathrm{h}^{-\frac{1}{2}}[v]\right\|_{\mathcal{F}}^{2}
$$

for all $v \in H^{1}(\mathcal{K})$. 


\subsection{Technical lemmas}

In this Section we recall some well known results. For the proofs we refer to [10].

Lemma 2.2 (Inverse inequality) Let $v_{h} \in V_{b s}$, then there holds

$$
c_{I}^{-1}\left\|\mathrm{~h}^{2} \Delta v_{h}\right\|_{\mathcal{K}} \leq\left\|\mathrm{h} \nabla v_{h}\right\|_{\mathcal{K}} \leq c_{I}\left\|v_{h}\right\|_{\mathcal{K}},
$$

where the constant $c_{I}>0$ is independent of $h$.

Lemma 2.3 (Trace inequality) Let $v \in\left[H^{1}(\mathcal{K})\right]^{m}$ and $v_{h} \in\left[V_{b s}\right]^{m}$ with $m \geq\{1, d\}$, then there holds

$$
\begin{aligned}
\|\{v\}\|_{\mathcal{F}}+\|[v]\|_{\mathcal{F}} & \leq c_{T}\left(\left\|\mathrm{~h}^{-\frac{1}{2}} v\right\|_{\mathcal{K}}+\left\|\mathrm{h}^{\frac{1}{2}} \nabla v\right\|_{\mathcal{K}}\right), \\
\left\|\left\{v_{h}\right\}\right\|_{\mathcal{F}}+\left\|\left[v_{h}\right]\right\|_{\mathcal{F}} & \leq c_{T}\left\|\mathrm{~h}^{-\frac{1}{2}} v_{h}\right\|_{\mathcal{K}},
\end{aligned}
$$

where the constant $c_{T}>0$ is independent of $h$.

\subsection{Special functions}

We denote by $\pi_{p}: L^{2}(\Omega) \rightarrow V_{h}^{p}$ the $L^{2}$-projection onto $V_{h}^{p}$ defined by

$$
\int_{\Omega} \pi_{p} v w_{h} d x=\int_{\Omega} v w_{h} d x \quad \forall w_{h} \in V_{h}^{p}
$$

The projection $\pi_{p}$ satisfies the following approximation result: Let $v \in H^{p+1}(\mathcal{K})$, then

$$
\left\|v-\pi_{p} v\right\|_{\mathcal{K}}+h\left\|\nabla\left(v-\pi_{p} v\right)\right\|_{\mathcal{K}} \leq c h^{p+1}|v|_{p+1, \mathcal{K}} .
$$

Finally, we present two functions, playing a role similar to that of a Fortin interpolant, which will be used in the following.

Lemma 2.4 Let $a_{h} \in V_{h}^{0}$ and $b_{h}, c_{h} \in W_{h}^{0}$ be fixed. Then, there exists a unique function $\phi_{h} \in V_{b s}$ such that

$$
\left\{\begin{aligned}
\pi_{0} \phi_{h} & =a_{h}, \\
\left.\left\{\nabla \phi_{h}\right\}_{s}\right|_{F} & =\left.b_{h}\right|_{F} \forall F \in \mathcal{F}, \\
{\left.\left[\nabla \phi_{h}\right]\right|_{F} } & =\left.c_{h}\right|_{F} \forall F \in \mathcal{F}_{i} .
\end{aligned}\right.
$$

Moreover $\phi_{h}$ satisfies the following stability result

$$
\left\|\mathrm{h}^{-1} \phi_{h}\right\|_{\mathcal{K}}^{2}+\left.\left\|\phi_{h}\right\|\right|^{2} \leq c\left(\left\|\mathrm{~h}^{-1} a_{h}\right\|_{\mathcal{K}}^{2}+\left\|\mathrm{h}^{\frac{1}{2}} b_{h}\right\|_{\mathcal{F}}^{2}+\left\|\mathrm{h}^{\frac{1}{2}} c_{h}\right\|_{\mathcal{F}_{i}}^{2}\right) .
$$

Proof Let us first establish the a priori estimate. Observe that

$$
\left\|\mathrm{h}^{-1} \phi_{h}\right\|_{\mathcal{K}} \leq\left\|\mathrm{h}^{-1} \pi_{0} \phi_{h}\right\|_{\mathcal{K}}+\left\|\mathrm{h}^{-1}\left(\phi_{h}-\pi_{0} \phi_{h}\right)\right\|_{\mathcal{K}} \leq\left\|\mathrm{h}^{-1} a_{h}\right\|_{\mathcal{K}}+c_{\star}\left\|\nabla \phi_{h}\right\|_{\mathcal{K}} .
$$


Then, invoking the trace inequality implies

$$
\|\| \phi_{h}\left\|^{2}=\right\| \mathrm{h}^{-\frac{1}{2}}\left[\phi_{h}\right]\left\|_{\mathcal{F}}^{2}+\right\| \nabla \phi_{h} \|_{\mathcal{K}}^{2} \leq c\left(\left\|\mathrm{~h}^{-1} a_{h}\right\|_{\mathcal{K}}^{2}+\left\|\nabla \phi_{h}\right\|_{\mathcal{K}}^{2}\right) .
$$

Thus it remains to estimate $\left\|\nabla \phi_{h}\right\|_{\mathcal{K}}$. Since $\left.\Delta \phi_{h}\right|_{\kappa} \in \mathbb{R}$ for all $\kappa \in \mathcal{K}$ and by integration by parts and (2.2) it follows that

$$
\begin{aligned}
\left\|\nabla \phi_{h}\right\|_{\mathcal{K}}^{2} & =-\left(\Delta \phi_{h}, \pi_{0} \phi_{h}\right) \mathcal{K}+\left(\left\{\nabla \phi_{h}\right\}_{s},\left[\phi_{h}\right]_{s}\right)_{\mathcal{F}}+\left(\left[\nabla \phi_{h}\right],\left\{\phi_{h}\right\}\right)_{\mathcal{F}_{i}} \\
& =\underbrace{-\left(\Delta \phi_{h}, a_{h}\right) \mathcal{K}}_{I}+\underbrace{\left(b_{h},\left[\phi_{h}\right]_{s}\right)_{\mathcal{F}}}_{I I}+\underbrace{\left(c_{h},\left\{\phi_{h}\right\}\right)_{\mathcal{F}}}_{I I I} .
\end{aligned}
$$

Applying the Cauchy-Schwarz, the inverse $(I)$ or the trace $(I I, I I I)$ for each term yields respectively

$$
\begin{gathered}
I \leq c_{I}\left\|\nabla \phi_{h}\right\|_{\mathcal{K}}\left\|\mathrm{h}^{-1} a_{h}\right\|_{\mathcal{K}} \\
I I \leq c_{T}\left\|\mathrm{~h}^{-1} \phi_{h}\right\|_{\mathcal{K}}\left\|\mathrm{h}^{\frac{1}{2}} b_{h}\right\|_{\mathcal{F}} \\
I I I \leq c_{T}\left\|\mathrm{~h}^{-1} \phi_{h}\right\|_{\mathcal{K}}\left\|\mathrm{h}^{\frac{1}{2}} c_{h}\right\|_{\mathcal{F}_{i}}
\end{gathered}
$$

and thus, applying Young's inequality and combining with (2.7), yields

$$
\begin{aligned}
& \left\|\nabla \phi_{h}\right\|_{\mathcal{K}}^{2} \\
& \leq c_{I}\left\|\nabla \phi_{h}\right\|_{\mathcal{K}}\left\|\mathrm{h}^{-1} a_{h}\right\|_{\mathcal{K}}+c_{T}\left\|\mathrm{~h}^{-1} \phi_{h}\right\|_{\mathcal{K}}\left(\left\|\mathrm{h}^{\frac{1}{2}} b_{h}\right\|_{\mathcal{F}}+\left\|\mathrm{h}^{\frac{1}{2}} c_{h}\right\|_{\mathcal{F}_{i}}\right) \\
& \leq \rho_{1}\left\|\nabla \phi_{h}\right\|_{\mathcal{K}}^{2}+c_{1}\left\|\mathrm{~h}^{-1} a_{h}\right\|_{\mathcal{K}}^{2}+\rho_{2}\left\|\mathrm{~h}^{-1} \phi_{h}\right\|_{\mathcal{K}}^{2}+c_{2}\left(\left\|\mathrm{~h}^{\frac{1}{2}} b_{h}\right\|_{\mathcal{F}}^{2}+\left\|\mathrm{h}^{\frac{1}{2}} c_{h}\right\|_{\mathcal{F}_{i}}^{2}\right) \\
& \leq\left(\rho_{1}+2 \rho_{2} c_{\star}\right)\left\|\nabla \phi_{h}\right\|_{\mathcal{K}}^{2}+\left(c_{1}+2 \rho_{2}\right)\left\|\mathrm{h}^{-1} a_{h}\right\|_{\mathcal{K}}^{2}+c_{2}\left(\left\|\mathrm{~h}^{\frac{1}{2}} b_{h}\right\|_{\mathcal{F}}^{2}+\left\|\mathrm{h}^{\frac{1}{2}} c_{h}\right\|_{\mathcal{F}_{i}}^{2}\right)
\end{aligned}
$$

for arbitrarily small $\rho_{1}, \rho_{2}>0$. Choosing $\rho_{1}$ and $\rho_{2}$ small enough leads then to the a priori estimate (2.6). To conclude the proof, it now suffices to observe that (2.5) is nothing more than a square linear system of size $N_{\mathcal{K}}+N_{\mathcal{F}}+N_{\mathcal{F}_{i}}$. Hence, existence and uniqueness of a solution of the linear system are equivalent. The a priori estimate (2.6) implies the uniqueness of the solution and hence the matrix is regular.

Observe that if the mesh is reduced to one single element $\kappa \in \mathcal{K}$, then the previous result simplifies to the following corollary.

Corollary 2.5 Fix $\kappa \in \mathcal{K}$ and let $a_{h} \in V_{h}^{0}(\kappa)$ and $b_{h} \in W_{h}^{0}(\kappa)$ be given. Then, there exists a unique function $\phi_{h} \in V_{b s}(\kappa)$ such that

$$
\left\{\begin{aligned}
\left.\pi_{0} \phi_{h}\right|_{\kappa} & =a_{h}, \\
\left.\nabla \phi_{h}\right|_{F} \cdot n_{\kappa} & =\left.b_{h}\right|_{F} \quad \forall F \in \mathcal{F}(\kappa) .
\end{aligned}\right.
$$

Moreover $\phi_{h}$ satisfies the following stability result

$$
\left.\left\|\mathrm{h}^{-1} \phi_{h}\right\|_{\kappa}^{2}+\mid\right] \phi_{h}\left[\left.\right|_{\kappa} ^{2} \leq c\left(\left\|\mathrm{~h}^{-1} a_{h}\right\|_{\kappa}^{2}+\left\|\mathrm{h}^{\frac{1}{2}} b_{h}\right\|_{\partial \kappa}^{2}\right) .\right.
$$




\section{Finite element methods for model problem}

In this section, we define all the methods that we will need in the further sections. We will then analyze how these methods are related to each other.

Crouzeix-Raviart method:

Find $u_{c r} \in V_{c r}$ such that

$$
\left(\nabla u_{c r}, \nabla v_{c r}\right)_{\mathcal{K}}=\left(f, v_{c r}\right)_{\mathcal{K}} \quad \forall v_{c r} \in V_{c r}
$$

We also will need the Crouzeix-Raviart solution using piecewise constant data: Find $u_{c r, 0} \in V_{c r}$ such that

$$
\left(\nabla u_{c r, 0}, \nabla v_{c r}\right)_{\mathcal{K}}=\left(\pi_{0} f, v_{c r}\right)_{\mathcal{K}} \quad \forall v_{c r} \in V_{c r}
$$

Raviart-Thomas method:

Find $\left(w_{r t}, u_{r t}^{0}\right) \in V_{r t} \times V_{h}^{0}$ such that

$$
\begin{aligned}
\left(w_{r t}, q_{r t}\right)_{\mathcal{K}} & +\left(u_{r t}^{0}, \nabla \cdot q_{r t}\right)_{\mathcal{K}}=0 \quad \forall q_{r t} \in V_{r t}, \\
& -\left(\nabla \cdot w_{r t}, v_{r t}^{0}\right)_{\mathcal{K}}=\left(f, v_{r t}^{0}\right)_{\mathcal{K}} \quad \forall v_{r t}^{0} \in V_{h}^{0} .
\end{aligned}
$$

BSDG-method:

Find $u_{b s} \in V_{b s}$ such that

$$
a\left(u_{b s}, v_{b s}\right)=\left(f, v_{b s}\right) \mathcal{K} \quad \forall v_{b s} \in V_{b s},
$$

with

$$
a(v, w)=(\nabla v, \nabla w)_{\mathcal{K}}-(\{\nabla v\},[w])_{\mathcal{F}}-([v],\{\nabla w\})_{\mathcal{F}}
$$

for all $v, w \in H^{2}(\mathcal{K})$.

In the following we shall point out some interesting properties of the BSDGmethod.

Remark 3.1 The discrete solution $u_{h}$ of (3.4) satisfies the following local mass conservation property

$$
-\int_{\partial \kappa}\left\{\nabla u_{h}\right\} \cdot n_{\kappa} d s=\int_{\kappa} f d x \quad \forall \kappa \in \mathcal{K}
$$

where $n_{\kappa}$ denotes the outer normal of element $\kappa$.

We now recall some results on the BSDG-approximation proved in [7].

Proposition 3.2 ([7, Proposition 2]) Let $u_{h}$ be the solution of (3.4). Then, the following estimate holds

$$
h\left\|f+\Delta u_{h}\right\|_{\mathcal{K}}+\left\|\mathrm{h}^{\frac{1}{2}}\left[\nabla u_{h}\right]\right\|_{\mathcal{F}_{i}}+\left\|\mathrm{h}^{-\frac{1}{2}} \overline{\left[u_{h}\right]}\right\|_{\mathcal{F}} \leq c h\left\|f-\pi_{0} f\right\|_{\mathcal{K}},
$$


and if $f \in H^{1}(\mathcal{K})$ there holds

$$
h\left\|f+\Delta u_{h}\right\|_{\mathcal{K}}+\left\|\mathrm{h}^{\frac{1}{2}}\left[\nabla u_{h}\right]\right\|_{\mathcal{F}_{i}}+\left\|\mathrm{h}^{-\frac{1}{2}} \overline{\left[u_{h}\right]}\right\|_{\mathcal{F}} \leq c h^{2}\|\nabla f\|_{\mathcal{K}} .
$$

Corollary 3.3 If $f$ is piecewise constant, i.e. $f \in V_{h}^{0}$, then

$$
\left\|\overline{\left[u_{h}\right]}\right\|_{\mathcal{F}}=0, \quad\left\|\left[\nabla u_{h}\right]\right\|_{\mathcal{F}_{i}}=0 \text { and }\left\|\Delta u-\Delta u_{h}\right\|_{\mathcal{K}}=0 .
$$

In the case of $f \in V_{h}^{0}$, we can further characterize the BSDG-approximation. To this aim, let us introduce the function satisfying the following conditions.

Lemma 3.4 ([7, Lemma 14]) Let $a_{h} \in V_{h}^{0}$ be fixed. Then, there exists a unique function $\phi_{h} \in V_{b s}$ such that

$$
\left\{\begin{aligned}
\Delta \phi_{h} & =a_{h}, \\
{\left.\left[\bar{\phi}_{h}\right]_{s}\right|_{F} } & =0 \quad \forall F \in \mathcal{F}, \\
{\left.\left[\nabla \phi_{h}\right]\right|_{F} } & =0 \quad \forall F \in \mathcal{F}_{i} .
\end{aligned}\right.
$$

Moreover $\phi_{h}$ satisfies the following stability result

$$
\left\|\phi_{h}\right\|^{2} \leq c\left\|a_{h}\right\|_{\mathcal{K}}^{2} .
$$

Corollary 3.5 If $f$ is piecewise constant, i.e. $f \in V_{h}^{0}$, then the solution $u_{h}$ of (3.4) is equal to the unique solution of the projection defined by Lemma 3.4 with $a_{h}=f$.

\section{Bubble stabilized DG with hanging nodes and edges}

In this section we will discuss stability of the BSDG-method on non-conforming meshes. We will therefore modify the definitions of the mesh quantities to allow for hanging nodes. These modifications are valid in this Section only. To analyse the BSDG-method on non-conforming meshes we must prove an inf-sup condition that holds uniformly independently of the mesh-geometry. This amounts to constructing a function in the finite element space that gives control of the jumps of the discrete solution. The existence of this function depends on the conformity of the mesh, in particular it is non-trivial to show that hanging-nodes are allowed. Here we will give sufficient conditions on the mesh for a bubble stabilized DG-method to be stable. Essentially we assume that the hanging nodes/edges appear due to the refinement of an underlying conforming mesh. Hence there is always one "refined" side and one "unrefined" side when there are hanging nodes/edges.

We redefine the set of interior faces by the set of all faces of all elements that are not included in the boundary $\partial \Omega$, i.e.

$$
\mathcal{F}_{i}=\{F \subset \partial \kappa \backslash \partial \Omega \mid F \text { is a face of } \kappa \text { for some } \kappa \in \mathcal{K}\},
$$

and in 3D we define the set of interior edges by

$$
\mathcal{E}_{i}=\{E \subset \partial \kappa \backslash \partial \Omega \mid E \text { is an edge of } \kappa \text { for some } \kappa \in \mathcal{K}\} .
$$


In $2 \mathrm{D}$ we define a hanging node as any vertex of the mesh that is contained in the interior of a face of $\mathcal{F}_{i}$. Analogously, in 3D a hanging edge is defined by an edge of $\mathcal{E}_{i}$ that is contained in the interior of a face of $\mathcal{F}_{i}$. If the spatial dimension is not specified we refer to a hanging $(d-2)$-subsimplex.

Our assumption on the mesh is then:

Assumption A1 Any face $F \in \mathcal{F}_{i}$ satisfies one of the following exclusive conditions:

(i) $F$ does not contain any faces in $\mathcal{F}_{i}$ other than $F$, and does not lie entirely in another face of $\mathcal{F}_{i}$.

(ii) $F$ lies entirely in another face $\tilde{F}$ of $\mathcal{F}_{i}$, and any other face that is not contained in $\tilde{F}$ sharing a hanging $(d-2)$-subsimplex with $F$ is of type (i).

(iii) $F$ contains $n_{F}>1$ faces of $\mathcal{F}_{i}$ other than $F$.

We denote the set of faces of type (i) by $\mathcal{F}_{c}$, those of type (ii) by $\mathcal{F}_{h e}$ and those of type (iii) by $\mathcal{F}_{n c}$. By definition $\mathcal{F}_{i}=\mathcal{F}_{c} \cup \mathcal{F}_{n c} \cup \mathcal{F}_{h e}$. For a given $F \in \mathcal{F}_{n c}$ the set $\mathcal{F}_{h e}(F)$ is defined as the set of associated faces contained in $F$ (with $F$ not included). Observe that any face of $\mathcal{F}_{h e}$ has at least one hanging $(d-2)$-subsimplex. An example of allowed hanging nodes in two dimensions is given in Fig. 1 and an example of disallowed hanging nodes is given in Fig. 2.

Now we introduce a modification of the average operator on faces with hanging $(d-2)$-subsimplices. For faces $F \in \mathcal{F}_{n c}$ we redefine the average operator by the one-sided value

$$
\left.\left\{\nabla u_{h}\right\}\right|_{F}=\left.\nabla u_{h}\right|_{\kappa_{1}}
$$

Fig. 1 Allowed non-conforming mesh in $2 \mathrm{D}$

Fig. 2 Mesh violating (Assumption A1) in 2D
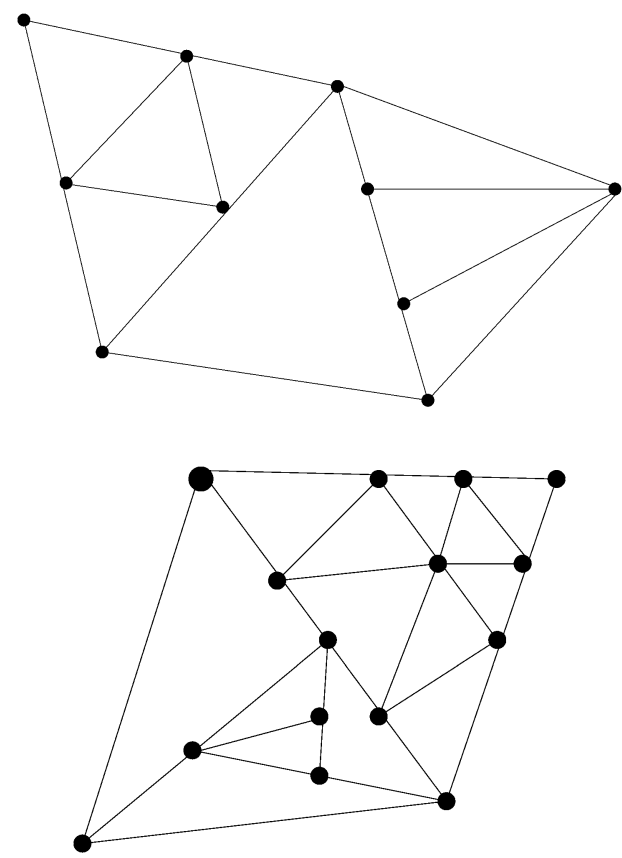
where $\kappa_{1}$ is the unrefined triangle, i.e. the one associated with the face of $F \in \mathcal{F}_{n c}$. We also define the operator giving the one-sided value from the other side compared to $\{\cdot\}$, for all $F \in \mathcal{F}_{n c}$ and for all $\tilde{F} \in \mathcal{F}_{h e}(F)$ we define

$$
\left.\left\langle u_{h}\right\rangle\right|_{\tilde{F}}=\left.u_{h}\right|_{\kappa_{2}}
$$

where $\kappa_{2}$ is the smaller simplex, i.e. on the refined side. On all faces in $\mathcal{F}_{c}$ the definition of the averages remain unchanged with equal weights $1 / 2,1 / 2$. It is easy to show that on a face with hanging $(d-2)$-subsimplices

$$
\left[\nabla u_{h} v_{h}\right]=\left[\nabla u_{h}\right]\left\langle v_{h}\right\rangle+\left\{\nabla u_{h}\right\}\left[v_{h}\right]
$$

Note that due to the hanging $(d-2)$-subsimplices the space $W_{h}^{0}$ is no longer uniquely defined. We therefore introduce

$$
\begin{aligned}
& \bar{W}_{h}^{0}=\left\{v_{h} \in L^{2}(\Gamma)\left|v_{h}\right|_{F} \in \mathbb{R}, \forall F \in \mathcal{F}_{c} \cup \mathcal{F}_{n c}\right\}, \\
& \underline{W}_{h}^{0}=\left\{v_{h} \in L^{2}(\Gamma)\left|v_{h}\right|_{F} \in \mathbb{R}, \forall F \in \mathcal{F}_{c} \cup \mathcal{F}_{h e}\right\},
\end{aligned}
$$

and observe that $\bar{W}_{h}^{0} \subset \underline{W}_{h}^{0}$. The $L^{2}$-projection onto $\bar{W}_{h}^{0}$ and $\underline{W}_{h}^{0}$ is denoted by $\bar{v}$ resp. $\underline{v}$ for any $v \in L^{2}(\Gamma)$. We then propose to show that if $\mathcal{T}_{h}$ is a mesh with hanging $(d-2)$-subsimplices, respecting Assumption $\mathrm{A} 1$, and $V_{b s}$ is the associated enriched DG-space there exists a function that allows to prove the inf-sup condition.

Lemma 4.1 Let $a_{h} \in V_{h}^{0}$ and $b_{h} \in \bar{W}_{h}^{0}$ and $c_{h} \in \underline{W}_{h}^{0}$ be fixed. Then, there exists a unique function $\phi_{h} \in V_{b s}$ such that

$$
\left\{\begin{aligned}
\pi_{0} \phi_{h} & =a_{h}, \\
\left.\left\{\nabla \phi_{h}\right\}_{s}\right|_{F} & =\left.b_{h}\right|_{F} \quad \forall F \in \mathcal{F}_{c} \cup \mathcal{F}_{n c} \cup \mathcal{F}_{e}, \\
\left.\underline{\left\langle\phi_{h}\right\rangle}\right|_{F} & =\left.c_{h}\right|_{F} \quad \forall F \in \mathcal{F}_{c} \cup \mathcal{F}_{h e}
\end{aligned}\right.
$$

where $\{\cdot\}_{s}$ is defined by the modified average operator of (4.1). Moreover $\phi_{h}$ satisfies the following stability result

$$
\left\|\mathrm{h}^{-1} \phi_{h}\right\|_{\mathcal{K}}^{2}+\|\| \phi_{h} \|^{2} \leq c\left(\left\|\mathrm{~h}^{-1} a_{h}\right\|_{\mathcal{K}}^{2}+\left\|\mathrm{h}^{\frac{1}{2}} b_{h}\right\|_{\mathcal{F}}^{2}+\left\|\mathrm{h}^{-\frac{1}{2}} c_{h}\right\|_{\mathcal{F}_{i}}^{2}\right) .
$$

Proof As in the proof of Lemma 2.4 we only need to show the bound for $\left\|\nabla \phi_{h}\right\|_{\mathcal{K}}$. In a similar fashion as for Lemma 2.4 we have

$$
\begin{aligned}
\left\|\nabla \phi_{h}\right\|_{\mathcal{K}}^{2} & =-\left(\Delta \phi_{h}, \pi_{0} \phi_{h}\right)_{\mathcal{K}}+\left(\left\{\nabla \phi_{h}\right\}_{s},\left[\phi_{h}\right]_{s}\right)_{\mathcal{F}}+\left(\left[\nabla \phi_{h}\right],\left\langle\phi_{h}\right\rangle\right)_{\mathcal{F}_{i}} \\
& =\underbrace{-\left(\Delta \phi_{h}, a_{h}\right)_{\mathcal{K}}}_{I}+\underbrace{\left(b_{h},\left[\phi_{h}\right]_{s}\right)_{\mathcal{F}}}_{I I}+\underbrace{\left(\left[\nabla \phi_{h}\right], c_{h}\right)_{\mathcal{F}_{i}}}_{I I I} .
\end{aligned}
$$

The terms $I$ and $I I$ are treated in a similar fashion as above and for the term $I I I$ we have by a trace inequality:

$$
I I I \leq c_{T}\left\|\nabla \phi_{h}\right\| \mathcal{K}\left\|\mathrm{h}^{-\frac{1}{2}} c_{h}\right\|_{\mathcal{F}_{i}}
$$


It remains to establish that the number of unknowns matches the number of equations. Recall that by the construction of the finite element space on each element we have $d+2$ degrees of freedom. This means one degree of freedom for each face of the simplex and one on the volume. First we observe that nothing has changed on faces without hanging $(d-2)$-subsimplices, i.e. two conditions per face. We note that the number of conditions on a given unrefined face $F \in \mathcal{F}_{n c}$ should equal the cardinality of $\mathcal{F}_{h e}(F)$ plus one since we have for each face on both side one degree of freedom to assign. On faces $F$ with $(d-2)$-subsimplices, there is one equation on the unrefined side and one condition for each face on the refined side, resulting in the cardinality of $\mathcal{F}_{h e}(F)$ plus one as expected. This means that every simplex has one condition per face and one on the volume, resulting in the expected $(d+2) \times N_{\mathcal{K}}$ equations. We conclude that the system is square.

The BSDG formulation on non-conforming meshes satisfying Assumption A1 is given by (3.4), but with the modified average on faces with $(d-2)$-subsimplices, given by (4.1). It is straightforward to show the following inf-sup condition using the technique developed in $[8,9]$.

$$
\left.\left\|u_{h}\right\|\right|_{\text {aux }} \leq c \sup _{w_{h} \in V_{b s}} \frac{a\left(u_{h}, w_{h}\right)}{\left.\left\|w_{h}\right\|\right|_{\text {aux }}}
$$

where the auxiliary norm is given by

$$
\|\| u_{h}\left\|\left.\right|_{\text {aux }} ^{2}=\right\| \mathrm{h}^{-\frac{1}{2}} \overline{\left[u_{h}\right]}\left\|_{\mathcal{F}_{c} \cup \mathcal{F}_{n c}}^{2}+\right\| \nabla u_{h} \|_{\mathcal{K}}^{2} .
$$

Now it only remains to show that $|\|\cdot\||_{\text {aux }}$ is a norm on $V_{b s}$. To this end, we would like to show a Poincaré inequality. This is similar to Lemma 4.1 in [8] for the case without hanging $(d-2)$-subsimplices. Note that on faces with hanging $(d-2)$-subsimplices, we only control the average of the jump over the whole face, as given on the unrefined side. This implies that we need to show that indeed the full jumps over each face in $\mathcal{F}_{h e}$ can be controlled. This is a consequence of the Assumption A1, indeed it follows that

$$
\left\|\mathrm{h}^{-\frac{1}{2}}\left[u_{h}\right]\right\|_{\mathcal{F}_{n c}}^{2}=\left\|\mathrm{h}^{-\frac{1}{2} \overline{\left[u_{h}\right]}}\right\|_{\mathcal{F}_{n c}}^{2}+\left\|\mathrm{h}^{-\frac{1}{2}}\left(\left[u_{h}\right]-\overline{\left[u_{h}\right]}\right)\right\|_{\mathcal{F}_{n c}}^{2} .
$$

For the second term of the right hand side we consider any face $\tilde{F} \in \mathcal{F}_{n c}$. Again, let $\kappa_{1}$ denote the simplex on the unrefined side. On the other side of the face we define by $\left\{\kappa_{2 i}\right\}$ the set of simplices consisting of all elements having at least one hanging $(d-2)$-subsimplex contained in $\tilde{F}$ as node/edge. Moreover we let $\mathcal{F}_{2}$ denote the set of faces such that $F=\partial \kappa_{2 i} \cap \partial \kappa_{2 j}$ for some $i, j$ with $i \neq j$. Note that all faces in $\mathcal{F}_{2}$ must be type (i) by Assumption A1. A scaling argument from a macro reference element including $\kappa_{1} \cup\left(\cup \kappa_{2 i}\right)$ we may deduce the upper bound

$$
\left\|\mathrm{h}^{-\frac{1}{2}}\left(\left[u_{h}\right]-\overline{\left[u_{h}\right]}\right)\right\|_{\tilde{F}}^{2} \leq c\left(\left\|\nabla u_{h}\right\|_{\kappa_{1}}^{2}+\sum_{i}\left\|\nabla u_{h}\right\|_{\kappa_{2 i}}^{2}+\left\|\mathrm{h}^{-\frac{1}{2}}\left[u_{h}\right]\right\|_{\mathcal{F}_{2}}^{2}\right) .
$$

To prove (4.6) we have decomposed the difference between the jump and its average in the contributions to the two sides. On the element $\kappa_{1}$ we use approximation and a 
trace inequality. On the refined side one may readily show that if the gradients in the elements $\kappa_{2 i}$ are all zero and the jumps in $\mathcal{F}_{2}$ are zero then the function is constant in $\bigcup \kappa_{2 i}$ and hence the trace minus its average on $\tilde{F}$ is zero. Hence (4.6) follows by scaling. Note that here we use the second part of Assumption A1(ii) to ensure that faces with hanging $(d-2)$-subsimplices can not be nested (see Fig. 2). Since all faces in $\mathcal{F}_{2}$ are type (i) may then conclude in a standard fashion, since

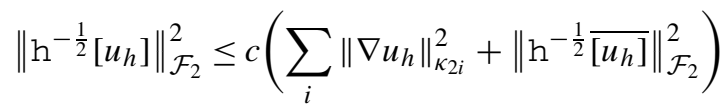

this yields the Poincaré inequality and thus norm equivalence

$$
c\left|\left\|u_{h}\right\|\right| \leq\left|\left\|u_{h}\right\|\right|_{\text {aux }} \leq\left|\left\|u_{h}\right\|\right| .
$$

Optimal a priori error estimates in the norm $\|\cdot\| \|$ and in the $L^{2}$-norm, now follows in the standard fashion.

Remark 4.2 The conditions of Assumption A1 are sufficient and can be relaxed. In particular, the second part of (ii) is unnecessarily strict. If it is relaxed, the above results may be proven using repeated use of (4.6). The constants in the above estimates will depend on the local quasi uniformity of the mesh in a non-trivial way and may degenerate as the number of levels increase (see the lower left element of Fig. 2.)

\section{Raviart-Thomas approximation as post-processed Crouzeix-Raviart solution}

In 1985, Marini [17] proposed an inexpensive method for the computation of the Raviart-Thomas approximation based on the Crouzeix-Raviart method in the particular case of $d=2$ and a elementwise constant $f \in V_{h}^{0}$. Let $u_{c r} \in V_{c r}$ be the CrouzeixRaviart solution defined by (3.1). Then, Marini proved that the Raviart-Thomas solution $\left(w_{r t}, u_{r t}^{0}\right) \in V_{r t} \times W_{h}^{0}$ of (3.3) is given by

$$
\begin{aligned}
& \tilde{w}_{r t, \kappa}=\nabla u_{c r}-\frac{f}{2}\left(x-\pi_{0} x\right), \\
& \tilde{u}_{r t, \kappa}^{0}=\pi_{0} u_{c r}-\frac{f}{4}\left(\left|\pi_{0} x\right|^{2}-\pi_{0}|x|^{2}\right) .
\end{aligned}
$$

In the following, we propose a generalization to that formula for $d=2,3$ and for general right hand sides. Denote by $u_{c r, 0} \in V_{c r}$ the solution of (3.2). On each element $\kappa \in \mathcal{K}$, find $\left(w_{r t, \kappa}, u_{r t, \kappa}^{0}\right) \in R T_{0}(\kappa) \times V_{h}^{0}(\kappa)$ such that

$$
\begin{aligned}
\left(w_{r t, \kappa}, q_{r t, \kappa}\right)_{\kappa} & +\left(u_{r t, \kappa}^{0}, \nabla \cdot q_{r t, \kappa}\right)_{\kappa}=\left(\bar{u}_{c r, 0}, q_{r t, \kappa} \cdot n_{\kappa}\right)_{\partial \kappa} \quad \forall q_{r t, \kappa} \in R T_{0}(\kappa) \\
& -\left(\nabla \cdot w_{r t, \kappa}, v_{r t, \kappa}^{0}\right)_{\kappa}=\left(f, v_{r t, \kappa}^{0}\right)_{\kappa} \quad \forall v_{r t, \kappa}^{0} \in V_{h}^{0}(\kappa)
\end{aligned}
$$

Lemma 5.1 The solution of (5.2) exists and is unique. 
Proof Since the left hand side of (5.2) is the Raviart-Thomas operator on a mesh consisting of one element, the solution of (5.2) exists and is unique.

Lemma 5.2 The global solution of (5.2) for all elements $\kappa \in \mathcal{K}$ is the RaviartThomas solution of (3.3).

Proof Denote by $\left(w_{r t}, u_{r t}^{0}\right) \in V_{r t} \times V_{h}^{0}$ the Raviart-Thomas solution of (3.3).

(i) Firstly, let us show that the solution $w_{r t, \kappa}$ lies in the global Raviart-Thomas space, i.e. $w_{r t, \kappa}$ has continuous normal flux over edges. To do so, define $w_{p}$ and $u_{p}^{0} \in V_{h}^{0}$ by

$$
\left.w_{p}\right|_{\kappa}=w_{r t, \kappa} \quad \text { resp. }\left.\quad u_{p}^{0}\right|_{\kappa}=u_{r t, \kappa}^{0} \quad \forall \kappa \in \mathcal{K} .
$$

We will see later that $w_{p} \in V_{r t}$, but so far only $\left.w_{p}\right|_{\kappa} \in V_{r t}(\kappa)$ for all $\kappa \in \mathcal{K}$ holds. Then, summing (5.2) over all elements, yields

$$
\begin{aligned}
\left(w_{p}, q_{r t}\right)_{\mathcal{K}} & +\left(u_{p}^{0}, \nabla \cdot q_{r t}\right)_{\mathcal{K}}=\left(\bar{u}_{c r, 0},\left[q_{r t}\right]\right)_{\mathcal{F}_{i}} \quad \forall q_{r t} \text { s.t. }\left.q_{r t}\right|_{\mathcal{K}} \in R T_{0}(\kappa), \\
& -\left(\nabla \cdot w_{p}, v_{r t}^{0}\right)_{\mathcal{K}}=\left(f, v_{r t}^{0}\right)_{\mathcal{K}} \quad \forall v_{r t}^{0} \in V_{h}^{0} .
\end{aligned}
$$

Now, test in (5.3) with $q_{r t}=\nabla v_{c}$ and $v_{r t}^{0}=\pi_{0} v_{c}$, for any $v_{c} \in V_{c r}$, which yields

$$
\begin{aligned}
\left(w_{p}, \nabla v_{c}\right)_{\mathcal{K}} & =\left(\bar{u}_{c r, 0},\left[\nabla v_{c}\right]\right)_{\mathcal{F}_{i}}, \\
-\left(\nabla \cdot w_{p}, v_{c}\right)_{\mathcal{K}} & =\left(f, \pi_{0} v_{c}\right)_{\mathcal{K}},
\end{aligned}
$$

since $\nabla \cdot w_{p} \in V_{h}^{0}$. Further, since $u_{c r}$ is the Crouzeix-Raviart solution (first equation) and by integration by parts (second equation) there holds equivalently

$$
\begin{aligned}
\left(w_{p}, \nabla v_{c}\right)_{\mathcal{K}} & =\left(\pi_{0} f, v_{c}\right)_{\mathcal{K}}, \\
\left(w_{p}, \nabla v_{c}\right)_{\mathcal{K}}-\left(\left[w_{p}\right], \bar{v}_{c}\right)_{\mathcal{F}_{i}} & =\left(f, \pi_{0} v_{c}\right)_{\mathcal{K}} .
\end{aligned}
$$

Subtracting now the first equation of (5.4) from the second one yields

$$
\left(\left[w_{p}\right], \bar{v}_{c}\right)_{\mathcal{F}_{i}}=0
$$

Now, choosing $v_{c} \in V_{c r}$ such that $\left.\bar{v}_{c}\right|_{F}=\left.\left[w_{p}\right]\right|_{F}$ for all $F \in \mathcal{F}_{i}$ yields that the normal jumps of $w_{p}$ are zero and thus $w_{p} \in V_{r t}$.

(ii) Let us now show that $\nabla \cdot w_{r t}=\nabla \cdot w_{p}$. Combining the second equation of (5.3) and (3.3) yields

$$
\left(\nabla \cdot\left(w_{r t}-w_{p}\right), v_{r t}^{0}\right)_{\mathcal{K}}=0, \quad \forall v_{r t}^{0} \in V_{h}^{0}
$$

Now choosing $v_{r t}^{0}=\nabla \cdot\left(w_{r t}-w_{p}\right)$ shows the desired result.

(iii) Further, we will show that $w_{r t}=w_{p}$. Observe that

$$
\left\|w_{r t}-w_{p}\right\|_{\mathcal{K}}^{2}=\left(w_{r t}, w_{r t}-w_{p}\right)_{\mathcal{K}}-\left(w_{p}, w_{r t}-w_{p}\right)_{\mathcal{K}}
$$


and by testing in the first equations of (5.3) and (3.3) with by testing in the $w_{r t}-w_{p} \in V_{r t}$ implies

$$
\left(w_{r t}, w_{r t}-w_{p}\right) \mathcal{K}=\left(w_{p}, w_{r t}-w_{p}\right) \mathcal{K}=0
$$

since $\nabla \cdot\left(w_{r t}-w_{p}\right)=0$ and $\left.\left[w_{r t}-w_{p}\right]\right|_{F}=0$ for all $F \in \mathcal{F}_{i}$. Thus we conclude that $w_{r t}=w_{p}$.

(iv) Finally we can conclude that $u_{r t}^{0}=u_{p}^{0}$. Indeed, by subtracting the first equation of (5.3) from the first one of (3.3) yields

$$
\left(u_{r t}^{0}-u_{p}^{0}, \nabla \cdot q_{r t}\right)_{\mathcal{K}}=0, \quad \forall q_{r t} \in V_{r t}
$$

since $w_{r t}=w_{p}$. Invoking another integration by parts and (2.2) implies that

$$
\left(\left[u_{r t}^{0}-u_{p}^{0}\right],\left\{q_{r t}\right\}\right)_{\mathcal{F}}=\left(\left[u_{r t}^{0}-u_{p}^{0}\right]_{s},\left\{q_{r t}\right\}_{s}\right)_{\mathcal{F}}=0
$$

and choosing $q_{r t} \in V_{r t}$ such that $\left.\left\{q_{r t}\right\}_{s}\right|_{F}=\left.\left[u_{r t}^{0}-u_{p}^{0}\right]_{s}\right|_{F} \in \mathbb{R}$ for all $F \in \mathcal{F}$ results in

$$
\left\|\left[u_{r t}^{0}-u_{p}^{0}\right]\right\|_{\mathcal{F}}=\left\|\left[u_{r t}^{0}-u_{p}^{0}\right]_{s}\right\|_{\mathcal{F}}=0 .
$$

Since $\|[\cdot]\|_{\mathcal{F}}$ is a norm on $V_{h}^{0}$ we conclude that $u_{r t}^{0}=u_{p}^{0}$.

One may now show that the result of [17] enters as a special case of Lemma 5.2. We omit the proof.

Lemma 5.3 In the particular case of $d=2$ and $f \in V_{h}^{0}$, the solution (5.1) coincides with the solution of (5.2).

\section{Bubble stabilized discontinuous Galerkin approximation as post-processed Crouzeix-Raviart solution}

In the previous Section we have seen a way to post-process the Crouzeix-Raviart solution in order to get the Raviart-Thomas solution. In a similar way we will derive a post-processing for the Crouzeix-Raviart solution to get a solution with superior local conservation properties in the space $V_{b s}$. It is not exactly the BSDG solution, but sufficiently close, with continuous normal flux over edges and preserving all other properties of the BSDG-method. This post-processing procedure will allow us to define a scheme for a DG-method that needs no more globally coupled degrees of freedom than the non-conforming Crouzeix-Raviart method.

Based on the Crouzeix-Raviart approximation $u_{c r}$ of (3.1) we post-process locally: for all $\kappa \in \mathcal{K}$, find $u_{p} \in V_{b s}(\kappa)$ such that

$$
a_{\kappa}\left(u_{p}, v_{h}\right)=\left(f, v_{h}\right)_{\kappa}-\left(u_{c r}, \nabla v_{h} \cdot n_{\kappa}\right)_{\partial \kappa} \quad \forall v_{h} \in V_{b s}(\kappa),
$$

with

$$
a_{\kappa}(v, w)=(\nabla v, \nabla w)_{\kappa}-\left(\nabla v \cdot n_{\kappa}, w\right)_{\partial \kappa}-\left(v, \nabla w \cdot n_{\kappa}\right)_{\partial \kappa} .
$$


Thus the approximation will satisfy the following local mass conservation property:

Remark 6.1 The discrete solution $u_{p}$ of (6.1) satisfies

$$
-\int_{\partial \kappa} \nabla u_{p} \cdot n_{\kappa} d s=\int_{\kappa} f d x \quad \forall \kappa \in \mathcal{K} .
$$

Lemma 6.2 (Local inf-sup condition) For all $u_{h} \in V_{b s}(\kappa)$, there holds

$$
\mid] u_{h}\left[\left.\right|_{\kappa} \leq c \sup _{v_{h} \in V_{b s}(\kappa)} \frac{a_{\kappa}\left(u_{h}, v_{h}\right)}{\mid] v_{h}\left[\left.\right|_{\kappa}\right.} .\right.
$$

Proof Since the bilinear form $a_{\kappa}(\cdot, \cdot)$ is nothing else than the bilinear form $a(\cdot, \cdot)$ from the BSDG-method (3.4) for a mesh consisting of one single element, the proof is similar to the stability proof of the BSDG-method [9] with some simplifications.

Lemma 6.3 (Stability of post-processing) The post-processing is well-posed, moreover the following stability estimate holds

$$
\mid] u_{p}\left[\left.\right|_{\kappa} \leq c\left(\|f\|_{\kappa}+\left\|h^{-\frac{1}{2}} u_{c r}\right\|_{\partial K}\right)\right.
$$

where $u_{c r}$ denotes the Crouzeix-Raviart solution of (3.1).

Proof This is a direct consequence of the previous inf-sup condition and the Poincaré inequality.

Proposition 6.4 Let $u_{p}$ be the post-processed Crouzeix-Raviart solution of (6.1). Then the normal flux of $u_{p}$ is continuous across interior faces, i.e.

$$
\left\|\left[\nabla u_{p}\right]\right\|_{\mathcal{F}_{i}}=0
$$

Proof Observe, using (3.1) and integration by parts, that

$$
0=\left(f, v_{c r}\right)_{\mathcal{K}}-\left(\nabla u_{c r}, \nabla v_{c r}\right)_{\mathcal{K}}=\left(f, v_{c r}\right)_{\mathcal{K}}-\left(\bar{u}_{c r},\left[\nabla v_{c r}\right]\right)_{\mathcal{F}_{i}}
$$

for all $v_{c r} \in V_{c r}$. Thus testing in (6.1) with $v_{c r} \in V_{c r}$ and summing over all elements yields

$$
-\left(\nabla u_{p}, \nabla v_{c r}\right)_{\mathcal{K}}+\left(\left[u_{p}\right],\left\{\nabla v_{c r}\right\}\right)_{\mathcal{F}}+\left(\left[\nabla u_{p}\right], \bar{v}_{c r}\right)_{\mathcal{F}_{i}}+\left(\left\{u_{p}\right\},\left[\nabla v_{c r}\right]\right)_{\mathcal{F}_{i}}=0
$$

Finally, another integration by parts yields

$$
\left(\left[\nabla u_{p}\right], \bar{v}_{c r}\right)_{\mathcal{F}_{i}}=0 .
$$

Now, choosing $v_{c r} \in V_{c r}$ such that

$$
\left.\bar{v}_{c r}\right|_{F}=\left.\left[\nabla u_{p}\right]\right|_{F} \quad \forall F \in \mathcal{F}_{i}
$$


implies that

$$
\left\|\left[\nabla u_{p}\right]\right\|_{\mathcal{F}_{i}}=0
$$

Similar as in Proposition 3.2 for the BSDG-solution of (3.4), the post-processed solution of (6.1) satisfies the following properties.

Proposition 6.5 Let $u_{p}$ be the solution of (6.1). Then, the following estimation holds

$$
h\left\|f+\Delta u_{p}\right\|_{\mathcal{K}}+\left\|\mathrm{h}^{-\frac{1}{2}}\left(\overline{\left\{u_{p}\right\}}-\bar{u}_{c r}\right)\right\|_{\mathcal{F}}+\left\|\mathrm{h}^{-\frac{1}{2}} \overline{\left[u_{p}\right]}\right\|_{\mathcal{F}} \leq c h\left\|f-\pi_{0} f\right\|_{\mathcal{K}},
$$

and if $f \in H^{1}(\mathcal{K})$ there holds

$$
h\left\|f+\Delta u_{p}\right\|_{\mathcal{K}}+\left\|\mathrm{h}^{-\frac{1}{2}}\left(\overline{\left\{u_{p}\right\}}-\bar{u}_{c r}\right)\right\|_{\mathcal{F}}+\left\|\mathrm{h}^{-\frac{1}{2}} \overline{\left[u_{p}\right]}\right\|_{\mathcal{F}} \leq c h^{2}\|\nabla f\|_{\mathcal{K}} .
$$

Proof By definition of $u_{p}$ we locally have

$$
0=\left(f, v_{h}\right)_{\kappa}-\left(\nabla u_{p}, \nabla v_{h}\right)_{\kappa}+\left(\nabla u_{p} \cdot n_{\kappa}, v_{h}\right)_{\partial \kappa}+\left(u_{p}-u_{c r}, \nabla v_{h} \cdot n_{\kappa}\right)_{\partial \kappa} .
$$

Summing over all elements leads to

$$
\begin{aligned}
0= & \left(f, v_{h}\right)_{\mathcal{K}}-\left(\nabla u_{p}, \nabla v_{h}\right)_{\mathcal{K}}+\left(\left\{\nabla u_{p}\right\},\left[v_{h}\right]\right)_{\mathcal{F}}+\left(\left[u_{p}\right],\left\{\nabla v_{h}\right\}\right)_{\mathcal{F}} \\
& +\left(\left[\nabla u_{p}\right],\left\{v_{h}\right\}\right)_{\mathcal{F}_{i}}+\left(\left\{u_{p}\right\}-\bar{u}_{c r},\left[\nabla v_{h}\right]\right)_{\mathcal{F}_{i}} .
\end{aligned}
$$

Now, using integration by parts we get that

$$
0=\left(f+\Delta u_{p}, v_{h}\right)_{\mathcal{K}}+\left(\overline{\left\{u_{p}\right\}}-\bar{u}_{c r},\left[\nabla v_{h}\right]\right)_{\mathcal{F}_{i}}+\left(\overline{\left[u_{p}\right]},\left\{\nabla v_{h}\right\}\right)_{\mathcal{F}} .
$$

Choosing $v_{h}=\phi_{h}$ as the projection defined by Lemma 2.4 with $a_{h}=\mathrm{h}^{2}\left(\pi_{0} f+\right.$ $\left.\Delta u_{p}\right), b_{h}=\mathrm{h}^{-1}\left(\overline{\left\{u_{p}\right\}}-\bar{u}_{c r}\right)$ and $c_{h}=\mathrm{h}^{-1} \overline{\left[u_{p}\right]}$ yields that

$$
\begin{aligned}
& \left\|\mathrm{h}\left(\pi_{0} f+\Delta u_{p}\right)\right\|_{\mathcal{K}}^{2}+\left\|\mathrm{h}^{-\frac{1}{2}}\left(\overline{\left\{u_{p}\right\}}-\bar{u}_{c r}\right)\right\|_{\mathcal{F}_{i}}^{2}+\left\|\mathrm{h}^{-\frac{1}{2}} \overline{\left[u_{p}\right]}\right\|_{\mathcal{F}}^{2} \\
& =\left(\pi_{0} f-f, \phi_{h}\right) \mathcal{K} \leq h\left\|\pi_{0} f-f\right\|_{\mathcal{K}}\left\|\mathrm{h}^{-1} \phi_{h}\right\|_{\mathcal{K}} \\
& \leq c h\left\|\pi_{0} f-f\right\|_{\mathcal{K}}\left(\left\|\mathrm{h}\left(\pi_{0} f+\Delta u_{p}\right)\right\|_{\mathcal{K}}^{2}+\left\|\mathrm{h}^{-\frac{1}{2}}\left(\overline{\left\{u_{p}\right\}}-\bar{u}_{c r}\right)\right\|_{\mathcal{F}_{i}}^{2}\right. \\
& \left.+\left\|\mathrm{h}^{-\frac{1}{2}} \overline{\left[u_{p}\right]}\right\|_{\mathcal{F}}^{2}\right)^{\frac{1}{2}}
\end{aligned}
$$

using the stability estimate (2.6). Therefore

$$
\left\|\mathrm{h}\left(\pi_{0} f+\Delta u_{p}\right)\right\|_{\mathcal{K}}+\left\|\mathrm{h}^{-\frac{1}{2}}\left(\overline{\left\{u_{p}\right\}}-\bar{u}_{c r}\right)\right\|_{\mathcal{F}_{i}}+\left\|\mathrm{h}^{-\frac{1}{2}} \overline{\left[u_{p}\right]}\right\|_{\mathcal{F}} \leq c h\left\|\pi_{0} f-f\right\|_{\mathcal{K}} .
$$

Finally observe that

$$
\left\|\mathrm{h}\left(f+\Delta u_{p}\right)\right\|_{\mathcal{K}} \leq\left\|\mathrm{h}\left(\pi_{0} f+\Delta u_{p}\right)\right\|_{\mathcal{K}}+h\left\|f-\pi_{0} f\right\|_{\mathcal{K}},
$$

which completes the result. 
Next, let us prove a lemma that will be helpful in the following proposition.

Lemma 6.6 Let $u_{p}$ be the post-processed Crouzeix-Raviart solution of (6.1) and $u_{h}$ be the BSDG solution of (3.4). Then there holds

$$
\left\|\pi_{0}\left(u_{h}-u_{p}\right)\right\|_{\mathcal{K}}=0 .
$$

Proof Let $v_{h} \in V_{b s}$ be the special function defined by Lemma 3.4 with $a_{h}=\pi_{0}\left(u_{h}-\right.$ $u_{p}$ ). Then, using equation (6.4) and integrating by parts yields

$$
-\left(u_{p}, \Delta v_{h}\right)_{\mathcal{K}}=\left(f, v_{h}\right)_{\mathcal{K}}
$$

since $\left[\nabla u_{p}\right]=0$ by Proposition 6.4. In a similar way, integrating the right hand side of (3.5) by parts we obtain

$$
-\left(u_{h}, \Delta v_{h}\right)_{\mathcal{K}}=\left(f, v_{h}\right)_{\mathcal{K}}
$$

and thus $\left\|\pi_{0}\left(u_{h}-u_{p}\right)\right\|_{\mathcal{K}}=0$.

Proposition 6.7 Let $u_{p}$ be the post-processed Crouzeix-Raviart solution of (6.1) and $u_{h}$ be the BSDG solution of (3.4). Then there holds

$$
\left\|u_{h}-u_{p}\right\|_{\mathcal{K}}+h\|\| u_{h}-u_{p}\left\|\leq C h^{2}\right\| f-\pi_{0} f \|_{\mathcal{K}}
$$

and

$\left\|\mathrm{h} \Delta\left(u_{h}-u_{p}\right)\right\|_{\mathcal{K}}+\left\|\mathrm{h}^{\frac{1}{2}}\left[\nabla\left(u_{h}-u_{p}\right)\right]\right\|_{\mathcal{F}_{i}}+\left\|\mathrm{h}^{-\frac{1}{2}} \overline{\left[u_{h}-u_{p}\right]}\right\|_{\mathcal{F}} \leq C h\left\|f-\pi_{0} f\right\|_{\mathcal{K}}$.

Proof Let $v_{h} \in V_{b s}$ and introduce the following auxiliary norm

$$
\left\|v_{h}\right\|_{r}^{2}:=\left\|\mathrm{h} \Delta v_{h}\right\|_{\mathcal{K}}^{2}+\left\|\mathrm{h}^{\frac{1}{2}}\left[\nabla v_{h}\right]\right\|_{\mathcal{F}_{i}}^{2}+\left\|\mathrm{h}^{-\frac{1}{2}} \overline{\left[v_{h}\right]}\right\|_{\mathcal{F}}^{2} .
$$

Then, using the triangle inequality, we immediately conclude by Propositions 3.2 and 6.5 that

$$
\|\| u_{h}-u_{p}\left\|\left.\right|_{r} \leq\left|\left\|u_{h}-u\right\|_{r}+\right|\right\| u-u_{p}\left\|_{r} \leq c h\right\| f-\pi_{0} f \|_{\mathcal{K}}
$$

where $u$ denotes the exact solution of (1.1). Therefore (6.6) holds. To prove (6.5), observe that by the zero average property of $u_{h}-u_{p}$ (Lemma 6.6) and (2.4) we have

$$
\left\|u_{h}-u_{p}\right\|_{\mathcal{K}} \leq \operatorname{ch}\left\|\nabla\left(u_{h}-u_{p}\right)\right\|_{\mathcal{K}} .
$$

On the other hand using the notation $\delta_{h}=u_{h}-u_{p}$ and proceeding similarly as in Lemma 2.4 we get

$$
\begin{aligned}
\left\|\nabla \delta_{h}\right\|_{\mathcal{K}}^{2} & =-\left(\Delta \delta_{h}, \delta_{h}\right)_{\mathcal{K}}+\left(\left[\nabla \delta_{h}\right],\left\{\delta_{h}\right\}\right)_{\mathcal{F}_{i}}+\left(\left\{\delta_{h}\right\},\left[\delta_{h}\right]\right)_{\mathcal{F}} \\
& \leq \rho_{1}\left\|\nabla \delta_{h}\right\|_{\mathcal{K}}+\rho_{2}\left\|\mathrm{~h}^{-1} \delta_{h}\right\|_{\mathcal{K}}^{2}+c \mid\left\|\delta_{h}\right\| \|_{r}
\end{aligned}
$$


Fig. 3 Commutative diagram of different numerical algorithms for the model problem (1.1) with piecewise constant source term $f$

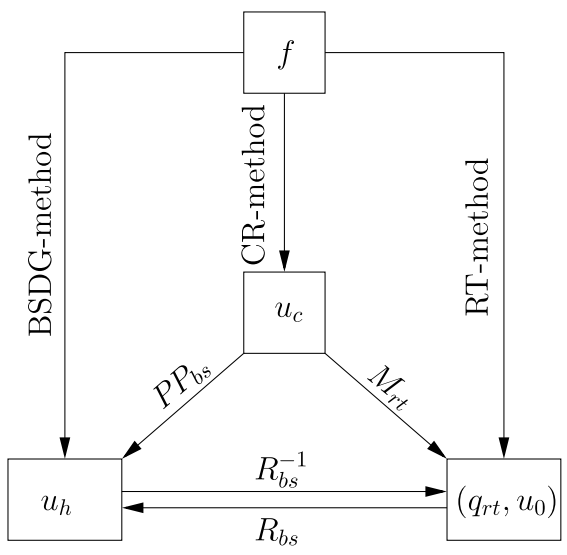

for any arbitrary small $\rho_{1}, \rho_{2}>0$. Invoking (6.8) and choosing $\rho_{1}, \rho_{2}$ sufficiently small yields

$$
\left\|\nabla\left(u_{h}-u_{p}\right)\right\|_{\mathcal{K}} \leq c \mid\left\|u_{h}-u_{p}\right\|_{r} .
$$

Now, respecting the trace and inverse inequalities combined with (6.8) then finally provides

$$
\left\|u_{h}-u_{p}\right\|_{\mathcal{K}}+h\left|\left\|u_{h}-u_{p}\right\|\right| \leq c h\left|\left\|u_{h}-u_{p}\right\|\right|_{r} .
$$

As consequence we can deduce the following theorem using the optimality of $u_{h}$.

Theorem 6.8 Let $u \in H^{2}(\Omega)$ be the exact solution of (1.1) and $u_{p}$ the post-processed Crouzeix-Raviart solution of (6.1). Then there holds

$$
\left\|u-u_{p}\right\|_{\mathcal{K}}+h\left|\left\|u-u_{p}\right\|\right| \leq c h^{2}\left(\|u\|_{2, \mathcal{K}}+\left\|f-\pi_{0} f\right\|_{\mathcal{K}}\right) .
$$

Proof Given the optimality of $u_{h}$, see [8, Theorems 7.4 and 7.5], i.e.

$$
\left\|u-u_{h}\right\|_{\mathcal{K}}+h\left|\left\|u-u_{h}\right\|\right| \leq c h^{2}\|u\|_{2, \mathcal{K}}
$$

we deduce the result by applying a triangle inequality and Proposition 6.7.

Thus, we finish this section by concluding that the post-processed solution $u_{p}$ of (6.1) shares all the properties of the BSDG solution. In addition to that $u_{p}$ has continuous normal fluxes over edges.

\section{Relation of finite element methods for piecewise constant data}

For piecewise constant data $f$, we will investigate the relation of the presented schemes and post-processing algorithms. 
To do that let us first introduce the following operator. Denote by $R_{b s}: V_{r t} \times V_{h}^{0} \rightarrow$ $V_{b s}$ the reconstruction operator defined such that

$$
\nabla R_{b s}\left(q_{r t}, v_{r t}^{0}\right)=q_{r t} \quad \text { and } \quad \pi_{0} R_{b s}\left(q_{r t}, v_{r t}^{0}\right)=v_{r t}^{0}
$$

for all $\left(q_{r t}, v_{r t}^{0}\right)$ such that $v_{r t}^{0} \in V_{h}^{0}$ and $\left.q_{r t}\right|_{\kappa} \in R T_{0}(\kappa)$ for all $\kappa \in \mathcal{K}$. The bijectivity of this operator is shown in Sect. 4.4 .3 of [7]. Its inverse is given by $\left(\nabla \cdot, \pi_{0} \cdot\right)$.

Then, denote by $u_{c}$ the Crouzeix-Raviart solution of (3.1) resp. (3.2), $u_{h} \in V_{b s}$ the BSDG solution (3.4), $u_{p} \in V_{b s}$ the post-processing defined by (6.1), $\left(w_{r t}, u_{r t}^{0}\right) \in$ $V_{r t} \times V_{h}^{0}$ the Raviart-Thomas solution (3.3) and $\left(w_{p}, u_{p}^{0}\right) \in V_{r t} \times V_{h}^{0}$ the postprocessing defined by (5.2).

Then we also introduce the two post-processing operators

$$
P P_{b s}: V_{c r} \rightarrow V_{b s} \quad \text { and } \quad M_{r t}: V_{c r} \rightarrow V_{r t} \times V_{h}^{0}
$$

such that $P P_{b s}\left(u_{c}\right)=u_{p}$ and $M_{r t}\left(u_{c}\right)=\left(w_{p}, u_{p}^{0}\right)$. Then assuming that the data $f$ is piecewise constant, i.e. $f \in V_{h}^{0}$, we conclude that

- $u_{h}=u_{p}$ by Proposition 6.7,

- $\left(w_{r t}, u_{r t}^{0}\right)=\left(w_{p}, u_{p}^{0}\right)$ by Lemma 5.2,

- $R_{b s}\left(w_{r t}, u_{r t}^{0}\right)=u_{h}$ and $R_{b s}^{-1} u_{h}=\left(w_{r t}, u_{r t}^{0}\right)$ by Lemma 20 of [7].

As consequence there holds

$$
P P_{b s}\left(u_{c}\right)=R_{b s}\left(M_{r t}\left(u_{c}\right)\right) \quad \text { and } \quad M_{r t}\left(u_{c}\right)=R_{b s}^{-1}\left(P P_{b s}\left(u_{c}\right)\right) .
$$

In other words, the diagram of Fig. 3 commutes.

Acknowledgements The authors thank Johnny Guzmán for the helpful and fruitful discussions and a knowledgeable reviewer for many helpful hints and corrections.

Open Access This article is distributed under the terms of the Creative Commons Attribution Noncommercial License which permits any noncommercial use, distribution, and reproduction in any medium, provided the original author(s) and source are credited.

\section{References}

1. Antonietti, P.F., Brezzi, F., Marini, L.D.: Bubble stabilization of discontinuous Galerkin methods. Comput. Methods Appl. Mech. Eng. 198(21-26), 1651-1659 (2009)

2. Arnold, D.N.: An interior penalty finite element method with discontinuous elements. SIAM J. Numer. Anal. 19(4), 742-760 (1982)

3. Baker, G.A.: Finite element methods for elliptic equations using nonconforming elements. Math. Comp. 31(137), 45-59 (1977)

4. Baumann, C.E., Oden, J.T.: A discontinuous $h p$-finite element method for convection-diffusion problems. Comput. Methods Appl. Mech. Eng. 175, 311-341 (1999)

5. Brezzi, F., Manzini, G., Marini, L.D., Pietra, P., Russo, A.: Discontinuous Galerkin approximations for elliptic problems. Numer. Methods Partial Differ. Equ. 16(4), 365-378 (2000)

6. Brezzi, F., Marini, L.D.: Bubble stabilization of discontinuous Galerkin methods. In: Advances in Numerical Mathematics. In Proc. International Conference on the Occasion of the 60th birthday of Y.A. Kuznetsov, pp. 25-36. (2006) 
7. Burman, E., Stamm, B.: Bubble stabilized discontinuous Galerkin method for parabolic and elliptic problems. Numer. Math. 116(2), 213-241 (2008)

8. Burman, E., Stamm, B.: Low order discontinuous Galerkin methods for second order elliptic problems. SIAM J. Numer. Anal. 47(1), 508-533 (2008)

9. Burman, E., Stamm, B.: Symmetric and non-symmetric discontinuous Galerkin methods stabilized using bubble enrichment. C. R. Math. Acad. Sci. Paris 346(1-2), 103-106 (2008)

10. Ciarlet, P.G.: The Finite Element Method for Elliptic Problems, vol. 40. Springer, Berlin (2002)

11. Cockburn, B., Shu, C.-W.: The local discontinuous Galerkin method for time-dependent convectiondiffusion systems. SIAM J. Numer. Anal. 35(6), 2440-2463 (1998)

12. de Dios, B.A., Zikatanov, L.: Uniformly convergent iterative methods for discontinuous Galerkin discretizations. J. Sci. Comput. 40(1-3), 4-36 (2009)

13. Douglas, J., Dupont, T.: Interior penalty procedures for elliptic and parabolic Galerkin methods. In: Lecture Notes in Phys., vol. 58, pp. 207-216 (1976)

14. Johnson, C., Pitkäranta, J.: An analysis of the discontinuous Galerkin method for a scalar hyperbolic equation. Math. Comp. 46(173), 1-26 (1986)

15. Larson, M.G., Niklasson, A.J.: Analysis of a nonsymmetric discontinuous Galerkin method for elliptic problems: stability and energy error estimates. SIAM J. Numer. Anal. 42(1), 252-264 (2004)

16. Lesaint, P., Raviart, P.-A.: On a finite element method for solving the neutron transport equation. Mathematical aspects of finite elements in partial differential equations. In: Proc. Sympos., Math. Res. Center, Univ. Wisconsin, Madison, 1974, pp. 89-123. Academic Press, New York (1974). Publication No. 33, Math. Res. Center. Univ. of Wisconsin-Madison, 65N30 (82.65 82.35)

17. Marini, L.D.: An inexpensive method for the evaluation of the solution of the lowest order RaviartThomas mixed method. SIAM J. Numer. Anal. 22(3), 493-496 (1985)

18. Nitsche, J.: Über ein Variationsprinzip zur Lösung von Dirichlet Problemen bei Verwendung von Teilräumen, die keinen Randbedingungen unterworfen sind. Abh. Math. Semin. Univ. Hamb. 36, 9$15(1971)$

19. Oden, J.T., Babuska, I., Baumann, C.E.: A discontinuous $h p$ finite element method for diffusion problems. J. Comput. Phys. 146(2), 491-519 (1998)

20. Reed, W.H., Hill, T.R.: Triangular mesh methods for the neutron transport equation. Technical Report LA-UR-73-479 (1973)

21. Rivière, B., Wheeler, M.F., Girault, V.: A priori error estimates for finite element methods based on discontinuous approximation spaces for elliptic problems. SIAM J. Numer. Anal. 39(3), 902-931 (2001) (electronic)

22. Wheeler, M.F.: An elliptic collocation-finite element method with interior penalties. SIAM J. Numer. Anal. 15(1), 152-161 (1978) 